\title{
Correction to: Factors predicting the recurrence of Epstein-Barr virus-associated hemophagocytic lymphohistiocytosis in children after treatment using the HLH-2004 protocol
}

\author{
Ryu Yanagisawa ${ }^{1,2,3} \cdot$ Kazuyuki Matsuda $^{4}$. Shouichi Ohga ${ }^{5} \cdot$ Hirokazu Kanegane $^{6} \cdot$ Akira Morimoto $^{7}$. \\ Yasuhiro Okamoto ${ }^{8}$. Akira Ohara ${ }^{9} \cdot K_{\text {Keitaro Fukushima }}{ }^{10} \cdot$ Manabu Sotomatsu $^{11} \cdot$ Keiko Nomura $^{12} \cdot$ Akiko M. Saito $^{3}$. \\ Keizo Horibe ${ }^{3}$ E Eiichi Ishii ${ }^{13} \cdot$ Yozo Nakazawa ${ }^{2}$ (])
}

Published online: 11 April 2019

(C) Japanese Society of Hematology 2019

\section{Correction to: International Journal of Hematology https://doi.org/10.1007/s12185-019-02612-2}

The correct name of the first author should be "Ryu Yanagisawa", and not "Ryu Yanagaisawa" as given in the original publication of the article.

The original article can be found online at https://doi.org/10.1007/ s12185-019-02612-2.

Yozo Nakazawa

yxnakaza@shinshu-u.ac.jp

1 Division of Blood Transfusion, Shinshu University Hospital, Matsumoto, Japan

2 Department of Pediatrics, Shinshu University School of Medicine, 3-1-1, Asahi, Matsumoto, Nagano 390-8621, Japan

3 Clinical Research Center, National Hospital Organization Nagoya Medical Center, Nagoya, Japan

4 Department of Clinical Laboratory Sciences, School of Health Sciences, Shinshu University, Matsumoto, Japan

5 Department of Pediatrics, Graduate School of Medical Sciences, Kyushu University, Fukuoka, Japan

6 Department of Child Health and Development, Graduate School of Medical and Dental Sciences, Tokyo Medical and Dental University, Tokyo, Japan
7 Department of Pediatrics, Jichi Medical University School of Medicine, Shimotsuke, Tochigi, Japan

8 Department of Pediatrics, Kagoshima University Graduate School of Medical and Dental Sciences, Kagoshima, Japan

9 Department of Pediatrics, Toho University, Tokyo, Japan

10 Department of Pediatrics, Dokkyo Medical University, Shimotsuga, Mibu, Japan

11 Department of Pediatrics, Gunma Children's Medical Center, Shibukawa, Japan

12 Department of Pediatrics, Graduate School of Medicine and Pharmaceutical Sciences, University of Toyama, Toyama, Japan

13 Department of Pediatrics, Ehime University Graduate School of Medicine, Toon, Japan 